\title{
The complex secondary metabolites in plant-environment interaction and evolutionary adaptation of tea plant for sustainable green food production
}

\author{
Jian Zhao ${ }^{1}$, Penghui $\mathrm{Li}^{1}$, and Xiao-Chun $\mathrm{Wan}^{1}$ \\ ${ }^{1}$ Anhui Agricultural University
}

May 5, 2020

\begin{abstract}
While most people know the rich flavors, pleasant tastes, and numerous health benefits of teas, various contaminations with chemicals for controling weeds, insects, microbial pathogens and other tea garden managements, become increasingly concerned on tea products. Meanwhile most of tea secondary metabolites concentrated in tea leaves, such as catechins, affeine, volatiles, saponins, and theanine, are synthesized primarily for defenses against both biotic and abiotic stresses. However, few use of these plant natural products for weed, pest and disease control by tea producers disabled the sustainable and safty production of teas. This is partly because the lacking of detailed information about biosynthesis, regulation, defense mechanism of these tea specialized metabolites due to chemical diversity and metabolic complexicity. An in-depth understanding of the biosynthesis, regulation, and defensive mechanisms of these specialized metabolites would greatly facilitate the employment of the innate immunity of tea plants to minimaize the use of other health-harmful chemicals. The review highlights relevant contexts of metabolic genomics of tea plants, so as to guaide future research on genetic improvement of tea plants for both safety and nutritional quality of teas.
\end{abstract}

\section{Hosted file}

Tea secondary metabolism and environmental interaction-JZhao.doc available at https: //authorea.com/users/296695/articles/425709-the-complex-secondary-metabolites-in-plantenvironment-interaction-and-evolutionary-adaptation-of-tea-plant-for-sustainable-greenfood-production 


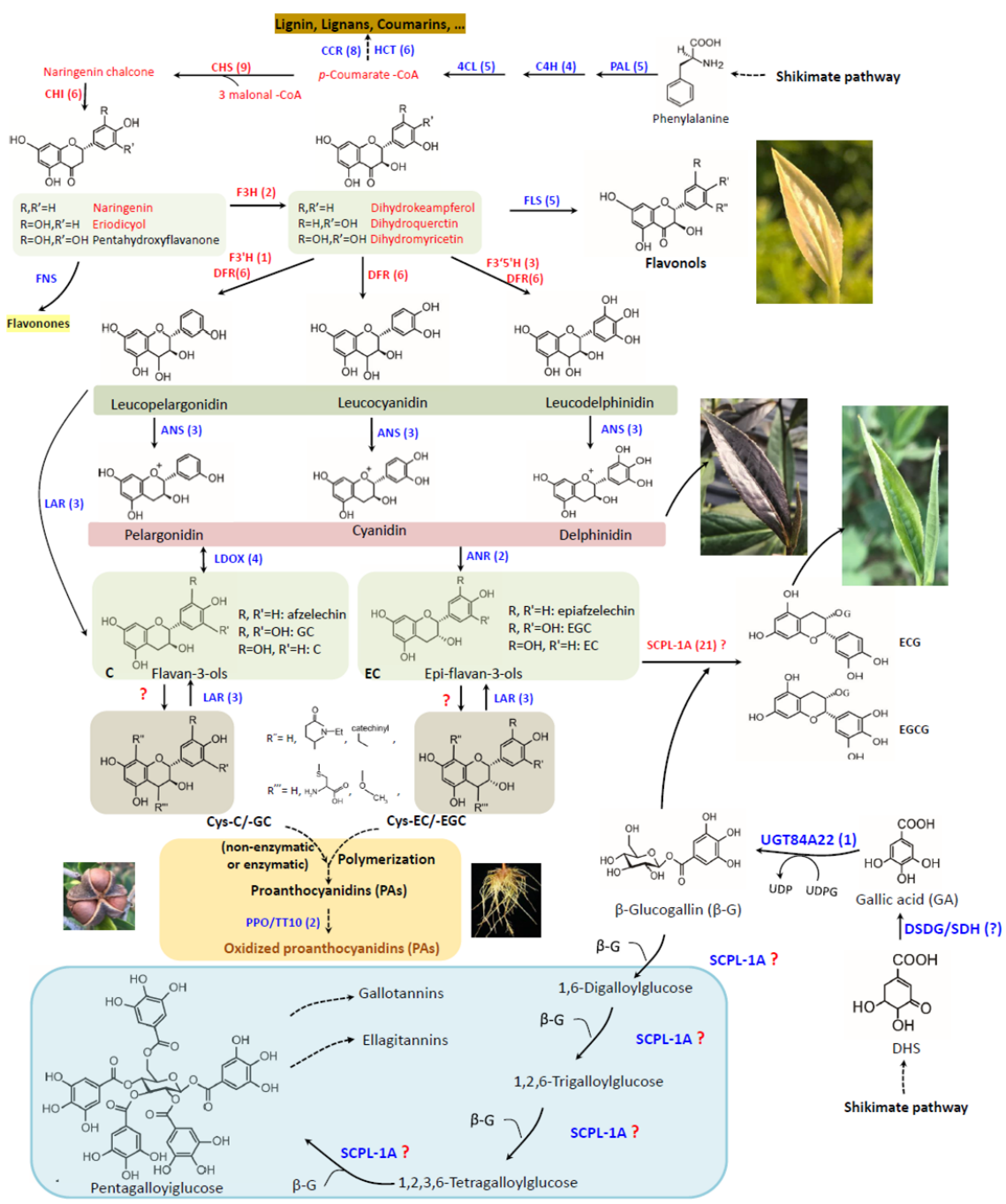



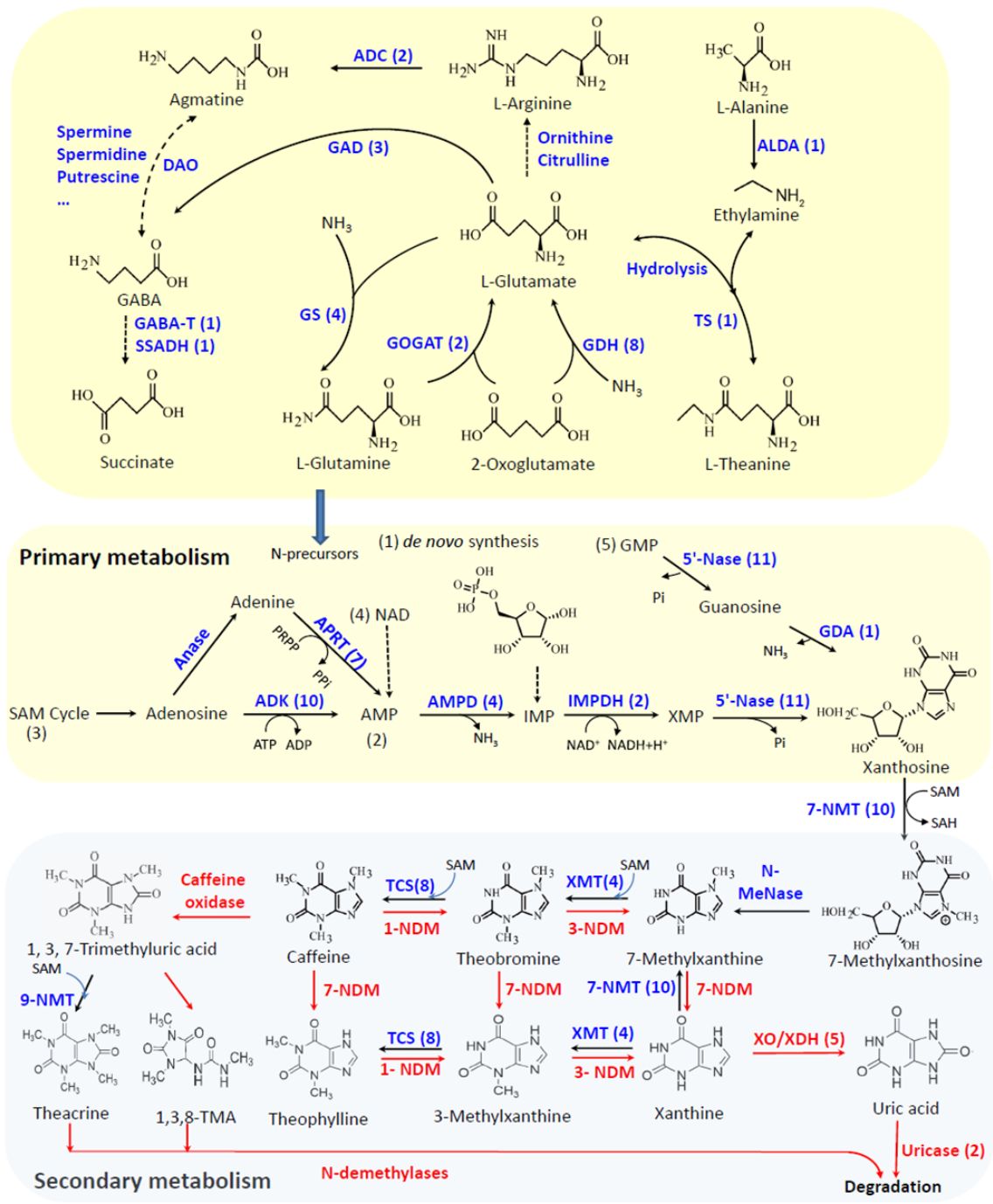

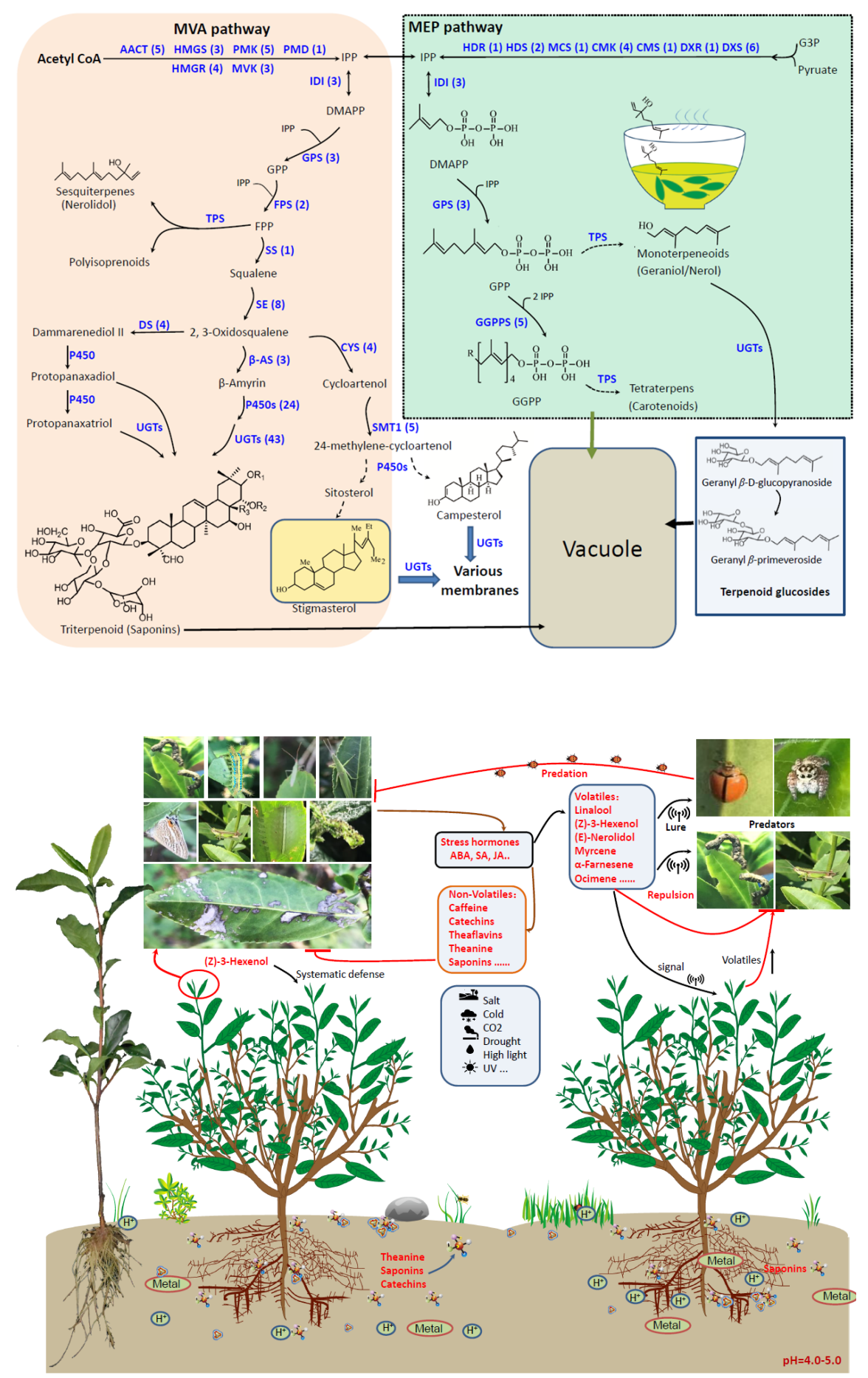

Secretion compunds (3) Germs Metal Metal ions $\mathbb{H}$ Hydrogen ions 


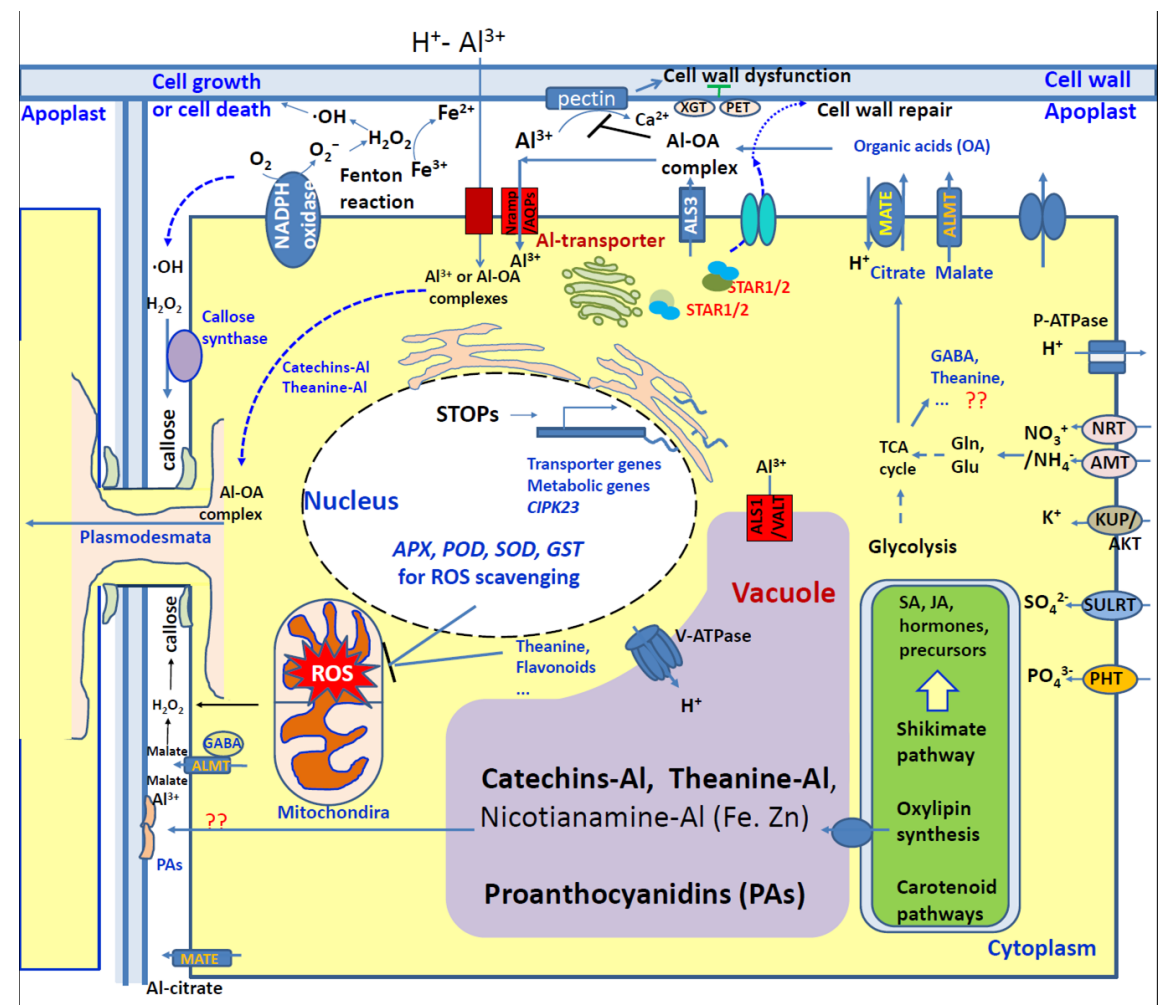

